\title{
LIDERAZGO VISIONARIO: CENTRO DEL CONOCIMIENTO
}

\author{
Por: \\ Rafael Pérez Uribe*
}

\section{ESUMEN}

El gestor del conocimiento es el ser humano. En el mundo como en las empresas es el centro de la destrucción y la creación del conocimiento. Por esta razón en las empresas se deben generar los escenarios adecuados para revertir el conocimiento de las personas y convertirlo en patrimonio de las organizaciones. El papel del líder es vital para la construcción de escenarios adecuados para que los trabajadores se conviertan en auténticos gestores del "knowledge» $y$ esto permita que las organizaciones sean entes permanentes de aprendizaje continuo.

\section{alabras clave}

Conocimiento, liderazgo, escenarios, rafting, cultura organizacional, valores, creencias.
$\mathrm{H}$ ablar de conocimiento es penetrar en el tema del ser humano y su relación con las empresas y organizaciones ${ }^{1}$ cualquiera sea su razón de ser, objeto social o finalidad. Más allá de resultados esperados en las empresas, como rentabilidad, eficacia, efectividad, competitividad, entre otros, el fin último de cualquier organización es satisfacer necesidades o en otras palabras, es la búsqueda de un mejor bienestar para el ser humano. De hecho el tema de gestión del conocimiento en el ámbito universal y sobre todo en el académico es tratado con extensión y profundidad por muchos autores. En la realidad empresarial, estos dos concep-tos, conocimiento y ser humano, se mueven mutuamente y de manera inseparable en cualquier organización, sobre todo la gestión del conocimiento, depende principalmente del paradigma que se maneje en la gestión compleja del ser humano.

Pero sigue siendo paradójico en el ámbito nacional e internacional, el hecho de las continuas olas de despidos individuales y

\footnotetext{
*Candidato a PhD Universidad de Nebrija, MSc.U de Quebec, Profesor Asociado Vicerrectoría de Investigación, Universidad EAN. Director del grupo de Investigaciones PyME.

1 En la literatura gerencial o de administración se maneja de manera indiscriminada el concepto de empresa y organización. Sin entrar en discusiones epistemológicas, en este escrito hablaremos de manera indiscriminada de los dos conceptos.
} 
masivos en muchas empresas tanto privadas como públicas ${ }^{2}$, sobre todo desde mediados de la década del 90 hasta la fecha de este escrito, como consecuencia de la búsqueda de unos mejores resultados empresariales. Para Bernardi y varias colegas (2000), un tema permanente de gran importancia para el éxito empresarial está constituido por una premisa fundamental: las personas son el eje central más valioso para cualquier empresa. Somos nosotros los que manejamos los recursos de diferentes formas. Construimos o destruimos permanentemente muchas de las variables que se manejan en el mundo y que inciden directa o indirectamente en las empresas: tecnología, información, conocimiento, economía, gobierno, relaciones entre países, política, influimos de manera positiva o negativa en el medio ambiente o en la responsabilidad social, etc.

Las condiciones sociales, económicas y culturales de este siglo hacen imprescindible que las empresas sean altamente creativas, innovadoras, se adapten ágilmente a los cambios, sobrevivan y crezcan, y para lograrlo necesariamente deben contar con una fuerza laboral comprometida, capacitada y motivada, que trabaje en busca del logro de la misión de la organización, pero que a la vez sienta que está logrando satisfacer sus deseos, expectativas y necesidades más profundas. Para ello se requiere de esfuerzo, tiempo, compromiso, habilidades, destrezas y conocimientos aportados por las personas que las constituyen.

Ahora bien, para comprender a una organización se torna necesario conocer a la "persona", que es aquel sistema complejo de por lo menos cinco dimensiones (sicológica, física, espiritual, intelectual y energética), compuesto además por factores internos y externos que están en continuo cambio y crecimiento. Los internos como capacidad de aprendizaje, motivación, percepción del ambiente, actitudes, entre otros. Los externos, como las recompensas, motivos sociales, relaciones de grupos, entre otros, son los que llevan a los individuos a ser o no, generadores de ideas, imaginativos, conflictivos, propulsores de cambios, luchadores. Estos complejos sistemas, que son los seres humanos de una organización y las no menos enmarañadas interrelaciones que entre ellos se entablan son el elemento esencial e imperceptible que determinará el futuro- exitoso o decadente- de la gestión del conocimiento en cada empresa.

La forma en que se gerencia al ser humano influye en la efectividad (eficiencia mas eficacia) del funcionamiento de la organización. Mucha de la literatura gerencial demuestra que algunas actitudes o estilos de gestión pueden aumentar el compromiso de los empleados y aumentar su productividad, de allí que sería importante pensar en la dirección de las personas como talentos valorables de la organización, que pueden ser medidos como resultado de la forma en que se dirige al personal. Si las personas son talentos valiosos para la organización, la dirección debería basar sus decisiones de gestión en un cálculo de costo-utilidad, es decir, sobre una estimación del costo y la utilidad implicados en una decisión. Si aceptamos las opiniones anteriores y reconocemos la relación entre rendimiento de los empleados y los estilos de gestión, las decisiones que toma la dirección tendrían que basarse en un correcto análisis de todos los factores: humanos y no humanos, implicados en la decisión a tomar.

1 Destruyendo muchas de ellas ???????????? 
Obligatoriamente, hay que hablar entonces del concepto de liderazgo para la generación de escenarios de construcción del conocimiento. Muchos conceptos se encuentran en la literatura, pero el que mas me ha impactado es el que se ha desarrollado por el grupo de investigación de Pymes $^{3}$ de la Universidad EAN: «Proceso de dirigir las actividades de los miembros de un grupo y de influir en ellas». Este concepto involucra varios aspectos: Personas a quien liderar, entraña una distribución desigual del poder entre los líderes y los miembros del grupo, la capacidad para usar las diferentes formas del poder para influir en la conducta de los seguidores y reconoce que el liderazgo es cuestión de valores.

Ahora bien el tema del liderazgo en el ámbito de la discusión en este articulo, toma una importancia vital para el éxito en la generación de escenarios adecuados para la construcción del conocimiento en el sentido que:1) un jefe desarrolle la capacidad para guiar y dirigir de manera visionaria, 2) una empresa puede tener una planeación adecuada, control y procedimiento de organización y no sobrevivir a la falta de un líder apropiado, 3) un buen liderazgo es vital para la supervivencia de cualquier negocio u organización y 4) Una organización con una planeación deficiente y malas técnicas de organización y control han sobrevivido debido a la presencia de un liderazgo dinámico.

\section{¿Pero que significa un liderazgo dinámico y visionario, generador de escenarios armónicos de trabajo? Es el que tiene las siguientes características:}

Es aquella (aquel) jefe que junto con sus colaboradores, revisa el panorama general de su proceso o unidad de trabajo, recopila las ideas, las reacciones y los compromisos de aquellos que poseen datos relevantes. Durante el proceso, formula y define conjuntamente las metas, los programas, las responsabilidades y los puntos de control con su gente. Después de haber discutido con ellos sus nuevas asignaciones, se mantiene en contacto con su gente para mostrarles su interés porque logren un buen desempeño. Les señala, tanto sus fuerzas como sus debilidades personales y brinda a cada quien la oportunidad de que aporte sus sugerencias para mejoras.

Este jefe - líder analiza las situaciones, aceptando lo que no funciona para su cambio $y /$ o transformación. No busca culpables y averigua en conjunto las causas de los problemas. De hecho refleja en sus actos del día a día: proactividad, innovación y creatividad haciéndole ver a los trabajadores la importancia del cambio. Da ejemplo de disciplina, responsabilidad, respeto por el ser humano, empoderamiento y tiene pleno conocimiento del proceso para adquirir capacidad de transmitirlo y hacer que el grupo se identifique y se comprometa con los objetivos corporativos.

De manera participativa crea una Visión, Misión y Objetivos que se puedan alcanzar compartiendo esfuerzos y trabajo en común. Sabe escuchar y comprender las ideas de sus colaboradores para alimentar y mejorar los procesos de cada área y así alcanzar los objetivos. Delega tareas importantes que permiten generar nuevas responsabilidades acrecentando la autoestima de todos.

Es una persona que trabaja fuertemente sin evadir responsabilidades, toma la iniciativa en la búsqueda de soluciones cuando se presentan problemas y enseña a su gente como un maestro, a mejorar cuando se comete un error. Reconoce las labores bien hechas y felicita

$\overline{3}$ Extraído de las discusiones del grupo. Es la primera vez que se publica este concepto. 
con objetividad a aquellos (as) que sobresalen en su trabajo.

Controla sus emociones, acepta sus errores y tiene el coraje de disculparse cuando se falla. Cree y confía firmemente en los principios y valores para poderlos transmitir.

Además de todo lo anterior esta permanentemente desarrollando en su gente una serie de atributos de liderazgo por medio de estas acciones:

1)Enseña y corrige con paciencia, 2) $\mathrm{Da}$ opiniones para que las personas participen y descubran que pueden hacer las cosas mejor, 3) Demuestra entusiasmo en todo lo relacionado con las metas de la organización, 4) Enseña a las personas a escuchar a los demás y aprender de cada experiencia vivida, 5) Hace un seguimiento del día a día para medir los resultados, 6) Crea espacios necesarios de manera individual y grupal para generar grandes cambios y un alto nivel de creatividad, 7) Irradia compromiso hacia el personal generando confianza y apoyo y entregándoles las herramientas necesarias para su desarrollo como líder y 8) Estimula a las personas para que tomen decisiones.

No es fácil ser jefe - líder, pero en la medida que haga practica todas estas condiciones, la generación, adaptación y asimilación de conocimiento por parte de su gente para la empresa será más fácil, potente y real. Aunque el tema de Liderazgo no se debe manejar de una sola forma y conociendo que existen diferentes enfoques, el que se presenta a continuación nace de una realidad vivida y discutida en varias empresas exitosas:

- Aclare responsabilidades a los inmediatos colaboradores. No asuma que su gente ya conoce lo que tiene que hacer, asegúrese que realmente lo sabe.

- Defina los límites para la toma de decisiones (delimitación sobre el que debe. hasta dónde puede y hasta dónde no)

- Analice en la toma de decisiones alternativas de solución no solo en lo relacionado a costos sino en todas las posibles variables implícitas

- Defina las políticas de calidad, dejando claro los lineamientos sobre los que debe trabajar todo el equipo de trabajo.

- Transmita sus mensajes de manera asertiva y con respeto, en las direcciones que se requieran:Haciaabajo-Directivos/colaboradores inmediatos; Hacia arriba-colaboradores/ superiores -; Horizontal - Directivos/Directivos o Áreas/Áreas -; Diagonal - Directivos de línea y funcionales/colaboradores de otras áreas -

- Utilice oportunamente las comunicaciones de manera pertinente y justa en el momento preciso. No deje que los chismes aumenten. De hecho no le crea a los chismes. Llame a las personas involucradas y confróntelas, antes de tomar una decisión.

- Realice Reuniones ejecutivas periódicas para la transmisión asertiva de la comunicación, teniendo cuidado del manejo de la agenda, el tiempo y que no se convierta en «reunionitis» (exceso de reuniones). No haga solamente reuniones de castigo. Peor aun no las haga para hacer relievanza en los errores.

- Defina con su gente o los involucrados planes de acción y determine indicadores de gestión. Realice seguimiento periódico y ajustes necesarios. Informe a su superior inmediato de los adelantos en la obtención de resultados. 
- Sea coherente en todo lo que usted piensa, dice y hace. DE EJEMPLO.

- Haga el esfuerzo en conocer algo más de sus colaboradores.

- Fortalezca y refuerce en su gente los aspectos positivos.

- Escuche a su gente y construya el futuro con ellos.

- Sea valiente para aceptar críticas de su gente o de otros colegas. Si las va a hacer, hágalas con respeto.

- Cierre las puertas a la cultura del mutuo elogio (cepillo) y a los celos para compartir información.

- Dé confianza a su gente, sin perder autoridad, para que pierdan el miedo a hablar.

- Genere los espacios de dialogo necesarios para que sus colaboradores presenten ideas innovadoras para mejoramiento del trabajo. Esta es una de la formas para que se rompa la rutina y el acomodamiento a ella.

Hay que recordar que lo anterior no es una receta, pero por más capacitación que los gerentes reciban, el anterior enfoque de liderazgo dinámico generador de una cultura para la construcción del conocimiento, depende directamente de su actitud en llevarlo a la realidad.

Con todo esto en mente, las verdaderas empresas gestoras del conocimiento, construyen progresivamente una cultura organizacional adecuada, entendiéndose este concepto como lo explica Denison (1991) «a los valores, las creencias y los principios fundamentales del sistema gerencial de una organización, así como el conjunto de procedimientos y conductas gerenciales que sirven de ejemplo y refuerzan dichos principios básicos». En palabras de Robbins (1999) «representa los valores centrales que comparten la mayoría de los miembros de la organización y es un sistema de significado compartido, que los individuos con antecedentes diferentes o en distintos niveles de la organización tienden a describir en términos similares».

Estas definiciones contienen varias palabras claves: actitudes, creencias y valores:

- Las actitudes: son hábitos de pensamiento que condicionan nuestras reacciones y conducta con respecto a la vida. Son afirmaciones evaluativas (favorables o desfavorables) respecto a lo que nos rodea. Además son aprendidas y por lo tanto son modificables.

- Las creencias: es aquello que las personas tiene por verdadero y correcto. Son los filtros predispuestos de nuestra forma de percibir el mundo que nos rodea. Junto con los valores, son las fuentes más importantes de la motivación y las que gobiernan al cerebro haciendo posible la acción y el comportamiento.

- Los valores: pautas morales que guían la actuación de los individuos, es la filosofía de la vida, es todo lo bueno y deseable, es todo lo que favorece el desarrollo y la realización del hombre como persona, es una cualidad o calidad, es algo digno de ser buscado por alguien.

En un alto porcentaje, como se dijo anteriormente, la cultura la genera el líder - jefe que este a cargo de una compañía, de acuerdo a sus actitudes, creencias y valores. Si es un (a) déspota, habrá miedo y rencor. Podría haber resultados a corto plazo, pero a un costo alto, 
por encima de la armonía de trabajo de un grupo de personas. En este ambiente, las personas estarán esperando la oportunidad de un trabajo digno y respetuoso, en otra empresa, por más necesidad de un salario que se tenga. El estrés será alto y la productividad a mediano y largo plazo baja. Si por el contrario es una (un) líder visionario, como se explico atrás, se construirá un escenario para la generación del conocimiento, de manera más natural y espontánea.

Se podría decir que se podrían generar varios tipos de cultura. En este artículo solo se hablaran de dos tipos, cada una identificando los extremos: La rafting y la selvática. Para describir cada una de ellas se utilizara, en el caso de la primera, el concepto puro del significado del rafting que «es un deporte de equipo, donde los tripulantes de la balsa reman y orientan su peso de manera coordinada para recorrer los rápidos de los ríos y mantenerse a flote». En este tipo de cultura, el jefe - Líder acompaña a su equipo hasta el final y su participación es evidente. Es la posición del líder visionario.
En la cultura selvática, el ambiente esta enrarecido o «húmedo». La gente esta en las reuniones con el jefe con miedo, de hecho nadie habla en ellas por temor a recibir comentarios ácidos. Se hacen notar los errores permanentemente y se patina en ellos hasta que interiormente la gente se enferma tarde que temprano. No hay coherencia entre lo que se predica y la realidad. Las preferencias por algunos trabajadores son visibles. La equidad se maneja de acuerdo a lo que dice el jefe. Los colaboradores en este tipo de cultura se vuelven «aguantadores». En este ambiente, es difícil generar una gestión de conocimiento con amplitud.

Para finalizar, dejo para reflexionar, varios aspectos: ¿Con que líder le gustaría trabajar? ¿En que ambiente se genera conocimiento con amplitud desmedida? Además dejo planteada varias hipótesis: 1) Liderazgo visionario genera una cultura rafting y mayor probabilidad de generación de conocimiento y 2) Liderazgo déspota genera una cultura selvática con mayor probabilidad de destrucción de conocimiento. 


\section{BiBLIOGRAFÍA}

BERNARDI, Andréa. BILBAO, Alicia. BOCCO, Mirta. FUNES Alejandro. MIAZZO, Cristián y ZANINI, Rozana. Consideraciones sobre los Recursos Humanos en la Gestión de las PYMES. VI congreso de la pequeña y mediana empresa: PYME 2000, hacia una mejor competitividad.
DENISON Daniel. Cultura Corporativa y Productividad Organizacional. Legis. 1991.

ROBBINS Stephen, Comportamiento organizacional. Prentice Hall. $8^{a}$ edición 1999.

http://www.interpatagonia.com/turismoaventura/rafting.html. Consultado en noviembre de 2006. 


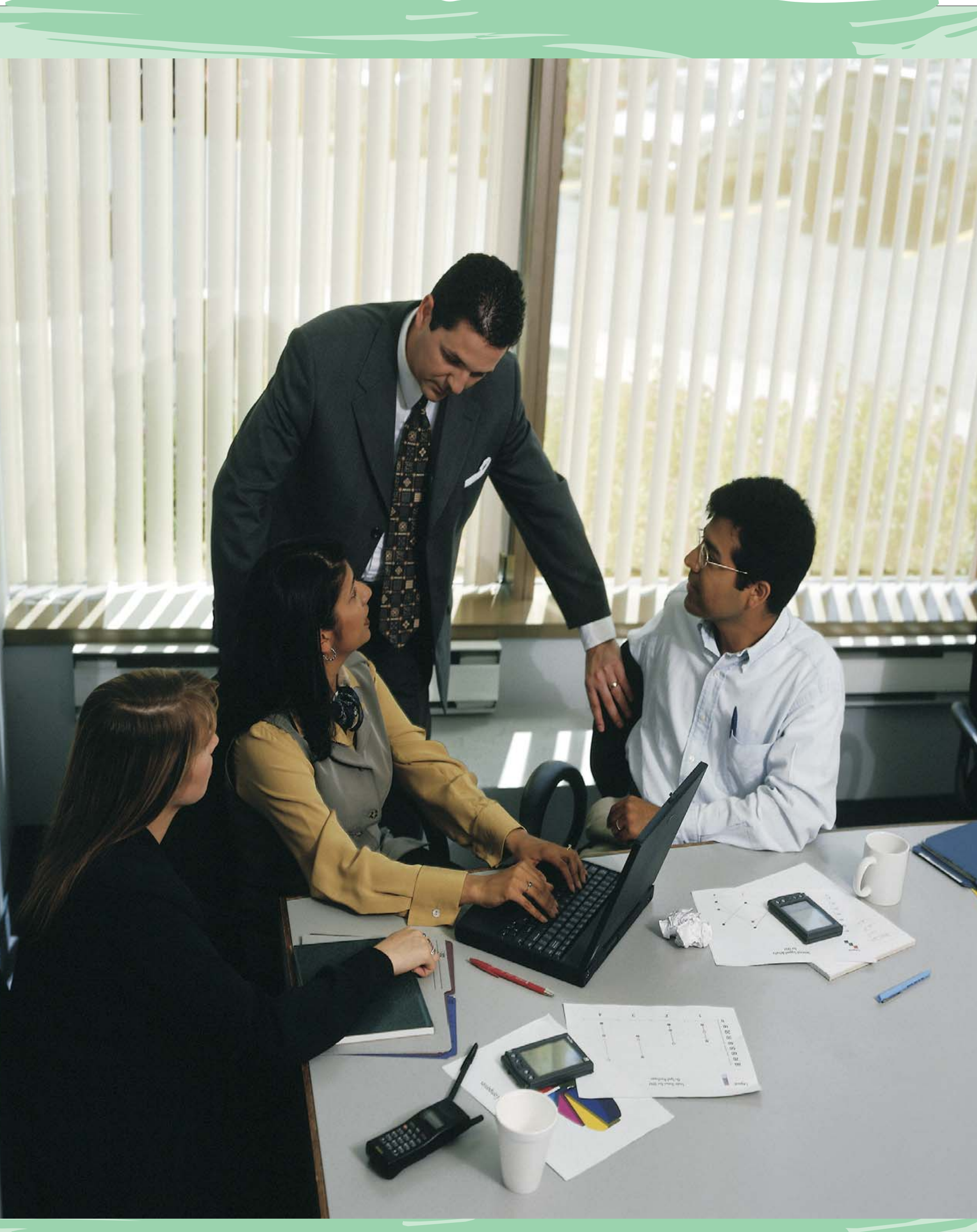

